\title{
A Novel Method for Estimating the Adaptive Ability of Guide Dogs Using Salivary $\operatorname{sIgA}$
}

\author{
Aya KIKKAWA ${ }^{1)}$, Yoshiko UCHIDA ${ }^{1) *}$, Yoshinori SUWA ${ }^{2)}$ and Kiyoshi TAGUCHI ${ }^{1)}$ \\ ${ }^{1)}$ School of Veterinary Medicine, Rakuno Gakuen University, 582 Bunkyodai-Midorimachi, Ebetsu 069-8501 and ${ }^{2)}$ Hokkaido Guide Dogs \\ for the Blind Association, 1-1 Minami30-Nishi8, Minamiku, Sapporo 005-8222, Japan
}

(Received 14 December 2004/Accepted 29 March 2005) ABSTRACT. Salivary secretory immunoglobulin A (sIgA) concentrations of prospective guide dogs for the blind were determined to clarify
whether salivary sIgA is useful in evaluating the potential suitability of guide dogs for the blind. Saliva was collected from 73 prospec-
tive guide dogs in the kennel on day 1 (the day of separation from volunteer puppy-raisers), $2,3,7$ and 14 during the estimation period
(at about 1 year old). We selected particularly suitable dogs (superior dogs) and unsuitable dogs (inferior dogs) on the basis of the train-
ers' estimation. All dogs were divided into two groups, those were acceptable dogs would become the guide dogs and rejected dogs
could not become guide dogs. The sIgA concentrations in superior dogs gradually increased from day 1 to 14 and those in inferior dogs
remained at low levels. Moreover, the sIgA concentrations on day 14 in the acceptable dogs were significantly higher than those in
rejected dogs. The cut-off point of sIgA concentrations on day 14 using an ROC curve was $90 \mathrm{EU} / \mathrm{ml}$, and the specificity of the esti-
mation at this point ( $70.4 \%$ ) was higher than that of trainers' estimation $(50 \%)$. Moreover, parallel testing using both trainers' estimation
and sIgA estimation, showed that specificity was further improved (79.5\%). The present study showed that sIgA concentration was
extremely useful in estimating the adaptive ability for guide dogs for the blind.

KEY WORDS: canine, guide dog, saliva, sIgA, stress.

J. Vet. Med. Sci. 67(7): 707-712, 2005

In Japan, 895 guide dogs are working and provide physical and mental support to the sight impaired. The raising of guide dogs is not easy, and the cost and labor involved is huge. Therefore, it is difficult to train more guide dogs, although many sight impaired need their own guide dogs.

Usually, prospective guide dogs are raised in the homes of volunteer puppy-workers from 7 weeks to 1-2 years old. After that, they return to the guide dogs associations and are estimated in terms of temperament for 1 week to 1 month (estimation period). Only the adaptive dogs are trained to become guide dogs for 1-2 years, including training with their users. They finish training and begin work as guide dogs when they are 2 years old. The appropriate method and time for the estimation of temperament is controversial, as most of the reasons for the rejection of guide dogs are related to behavioral problems rather than physical problems $[5,17,22]$.

With regard to the estimation period, most guide dogs associations estimated prospective dogs' behavior by several trainers. However, in many cases, the results from the various trainers were inconsistent due to the subjectivity of the trainers' estimation and the variations in the dogs' behavior in response to environmental stimuli. Therefore, most dogs thought to have potential as guide dogs are trained, and are re-estimated during the training period. In consequence, the dogs that subsequently fail to qualify as guide dogs are handed over to alternative homes at 2 years or older. As one of the prime reasons why the prospective guide dogs are rejected is nervousness and fearfulness $[3,5$,

\footnotetext{
* Correspondence to: Uchida, Y., School of Veterinary Medicine, Rakuno Gakuen University, 582 Bunkyodai- Midorimachi, Ebetsu 069-8501, Japan.
}

$16,17]$, it is more difficult to adapt to a new environment for rejected adult dogs than for younger animals. The problem of animal welfare and cost and labor efficiency would be improved, if the adaptive ability of guide dogs for the blind could be estimated objectively at an earlier age.

Recently, salivary sIgA concentration has been shown to be changed by psychological stresses in humans $[1,2,6,7$, $9,11,12,19-21,23]$, and the mechanism of this response was suggested as sympathetic-adrenal-medullary activation $[15,21]$. As samples are easily obtained without causing undue distress to the subject and only a little volume of sample to determine is required, salivary sIgA has been applied as an the ideal markers for psychological disorders [14], quality of life [4, 7], and personality [13] in humans. In dogs, previous study has suggested salivary sIgA to be useful as a stress marker [10]. In addition, Skandakumar et al. showed that adaptive ability in police dogs was associated with sIgA levels [18]. This gives rise to the view that sIgA concentrations might also be of use in estimating potential guide dogs for the blind and other service dogs.

In the present study, to clarify the efficacy of salivary sIgA in estimating guide dogs for the blind, we practically determined $\operatorname{sigA}$ concentrations in prospective guide dogs during their raising. Moreover, we also examined practical screening method using salivary sIgA.

\section{MATERIALS AND METHOD}

Study 1. Correlation between sIgA concentration during the estimation period and adaptive ability as guide dogs

Animals: Seventy-three healthy prospective guide dogs (35 male and 38 female) from the Hokkaido Guide Dogs for 
the Blind Association in Japan were used. The dogs were either Labrador Retrievers or Labrador $\times$ Golden Retriever crosses. These were 11 to 14 months old, after puppy walking, and were housed individually in a well-ventilated kennel $(1 \times 1.2 \mathrm{~m})$ of the association for estimation of their behavior and temperament for about 14 days (estimation period).

Saliva sampling and care: Saliva was collected on day 1 (the day of separation from volunteer puppy-raisers), 2, 3, 7 and 14 during the estimation period. The time of saliva sampling was between $1,230 \mathrm{hr}$ and $1,300 \mathrm{hr}$ on day 1 , and between $0900 \mathrm{hr}$ and $0930 \mathrm{hr}$ on the other days. Food was given at $0700 \mathrm{hr}$ and water was provided ad libitum, though the day of sampling, all animals were not allowed to drink water for $1 \mathrm{hr}$ before each sampling

To collect the saliva, cotton swabs, approximately $1 \mathrm{~cm}$ in diameter, were placed bilaterally in each dog's cheek pouches and left for $1 \mathrm{~min}$, with the handler gently holding the muzzle to prevent swallowing. Upon removal, the pair of absorbent cotton swabs was immediately placed in a pipette tip $(5 \mathrm{ml})$, and that was put in the centrifugation tube. The tube was covered and kept in a cooler box for about one hour during delivered to our laboratory. The saliva was extracted by centrifugation at $2,500 \mathrm{~g}$ for $30 \mathrm{~min}$ at $4{ }^{\circ} \mathrm{C}$. The saliva samples were stored at $-20^{\circ} \mathrm{C}$ until analysis.

Determination of SIgA concentration: Salivary sIgA concentration was determined by sandwich ELISA with a quantitation kit (Dog IgA Quantitation kit, Bethyl, Texas). Results are expressed in ELISA units (EU), where the concentration of the reference serum $(1 \mathrm{mg} / \mathrm{m} l)$ was defined as $100 \mathrm{EU} / \mathrm{ml}$. Six different saliva samples were analyzed, with 20 repeats each, and the inter-assay variation was between $4.3 \%$ and $10.8 \%$ (mean $7.2 \%$ ).

Estimation of temperament (Trainers' test): Three trainers walked with the prospective guide dogs on the street and in a park, and estimated the dogs' behavior and temperament. They also observed the dogs' behavior in the kennel. After the estimation period, the dogs were divided into two groups; those considered suitable and those thought to be unsuitable. The unsuitable dogs could not be trained as guide dogs, and they were given to volunteers.

We chose the superior dogs that all three trainers estimated as adaptive, and the inferior dogs which were estimated as unsuitable by all trainers.

Determination of guide dog ability: The rejected dogs were these regarded as unsuitable during the estimation period and the dogs which could not adapt to the guide dog training. The acceptable dogs were those that could adapt to all procedures necessary for guide dogs.

Statistical analysis: To evaluate variations of sIgA concentration in acceptable dogs or rejected dogs from day 1 to day 14, repeated- measure ANOVA was used.

To estimate differences in SIgA concentration between acceptable dogs and rejected dogs on each sampling day, student's $t$-test was used.

$P<0.05$ was considered significant.

To estimate the efficiency of the trainer's test, sensitivity, specificity, positive predictive value, negative predictive value, accuracy, and diagnostic efficiency were calculated.

To establish the cut-off point of SIgA concentration on day 14 , a receiver operating characteristics curve (ROC curve) was used. Estimation of the efficiency of the estimation of adaptive ability using sIgA concentrations ( $\operatorname{sgA}$ test), sensitivity, specificity, positive predictive value, negative predictive value, accuracy, likelihood ratio and diagnostic efficiency were calculated.

To estimate the efficacy of the trainer's test and $\operatorname{sigA}$ test conducted simultaneously (parallel test), sensitivity, specificity, positive predictive value, negative predictive value, accuracy, and diagnostic efficiency were calculated. The parallel test gave priority to specificity, and unsuitable dogs estimated by whichever test were taken to be unsuitable.

Study 2. Correlation between sIgA concentration in puppy period and adaptive ability for guide dogs

Animals: Twenty-six of the healthy prospective guide dogs (15 male and 11 female) from Hokkaido Guide Dogs for the Blind Association in Japan that were used in study 1 were used. These dogs were cared for by volunteer puppyworkers in their homes.

Saliva sampling and $\operatorname{sIg} A$ concentrations: Saliva was collected when the prospective guide dogs were 8 weeks $(8$ W), 6 months $(6 \mathrm{M})$, and 12 months $(12 \mathrm{M})$ old. After explanation of the method for saliva sampling (follow the method on study 1), saliva was collected by each puppyworker between $0630 \mathrm{hr}$ and $0730 \mathrm{hr}$ before walking and feeding. The centrifugation tubes containing the cotton swabs were stored at $4^{\circ} \mathrm{C}$ in puppy-workers' refrigerator. The saliva was extracted by centrifugation at $2500 \mathrm{~g}$ for 30 min at $4^{\circ} \mathrm{C}$ within $8 \mathrm{hr}$ and stored at $-20^{\circ} \mathrm{C}$ until analysis. The determination of salivary $\operatorname{sIgA}$ concentration followed

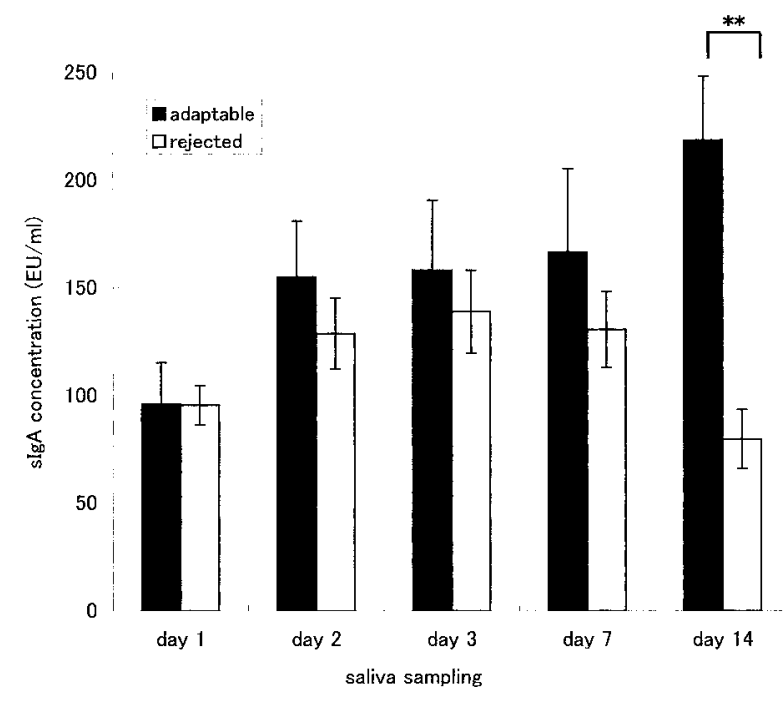

Fig. 1. The sIgA concentration in acceptable dogs $(n=25)$ or rejected dogs $(n=44)$ during the estimation period (average \pm $\mathrm{SE})$. A significant difference was shown on day $14(* *$ $\mathrm{P}<0.001)$. 

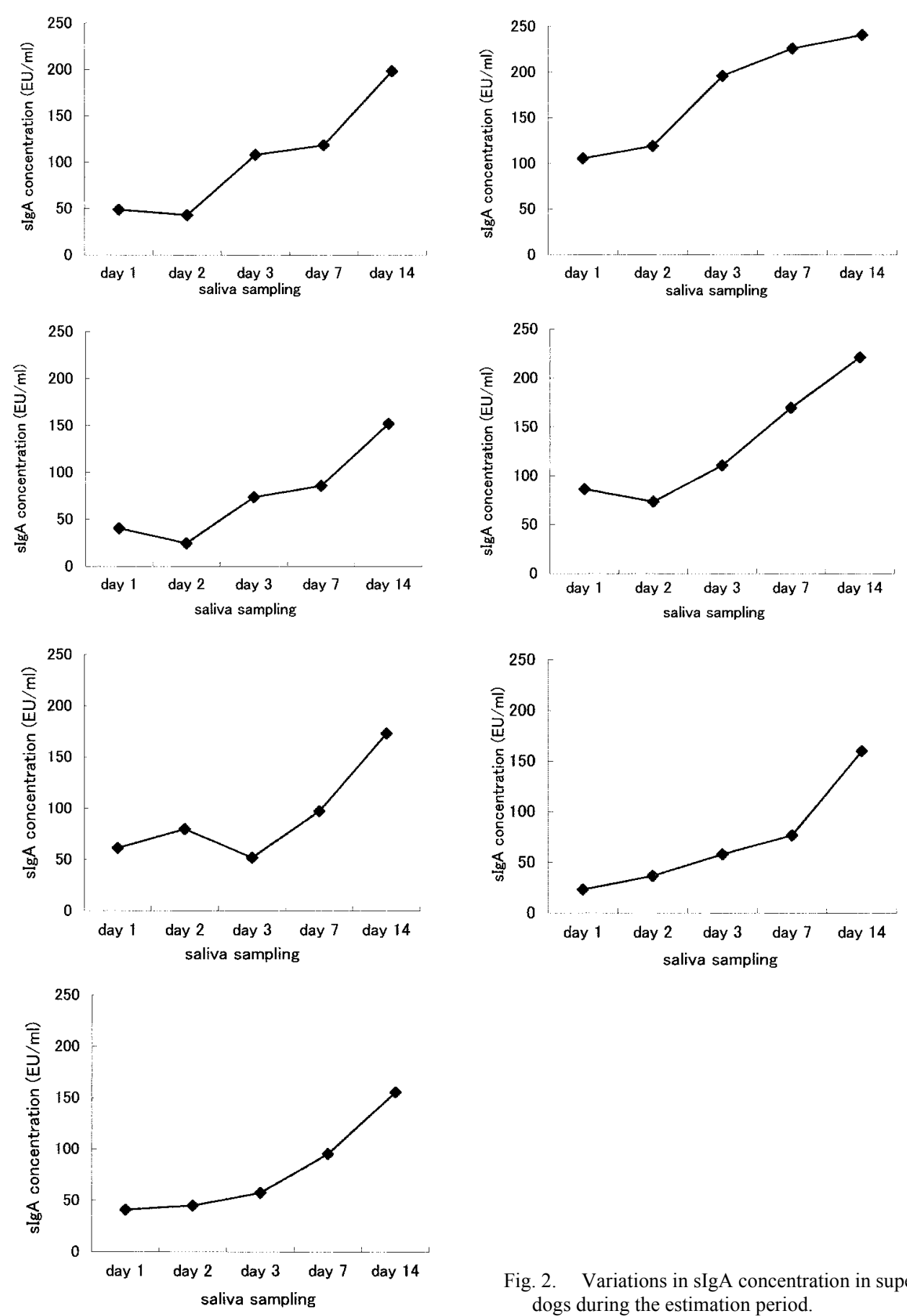

the procedure used in study 1 .

Statistical analysis: To estimate differences in sIgA concentration between acceptable dogs and rejected dogs for each sampling, student's $t$-test was used.

$P<0.05$ was considered significant.

Fig. 2. Variations in sIgA concentration in superior dogs during the estimation period.

\section{RESULTS}

Study 1. Correlation between SIgA concentration during the estimation period and adaptive ability as guide dogs.

During the estimation period, sufficient saliva could be collected from 69 dogs $(94.52 \%)$. Among these dogs, 25 dogs were acceptable (36\%), 44 were rejected (64\%), 7 

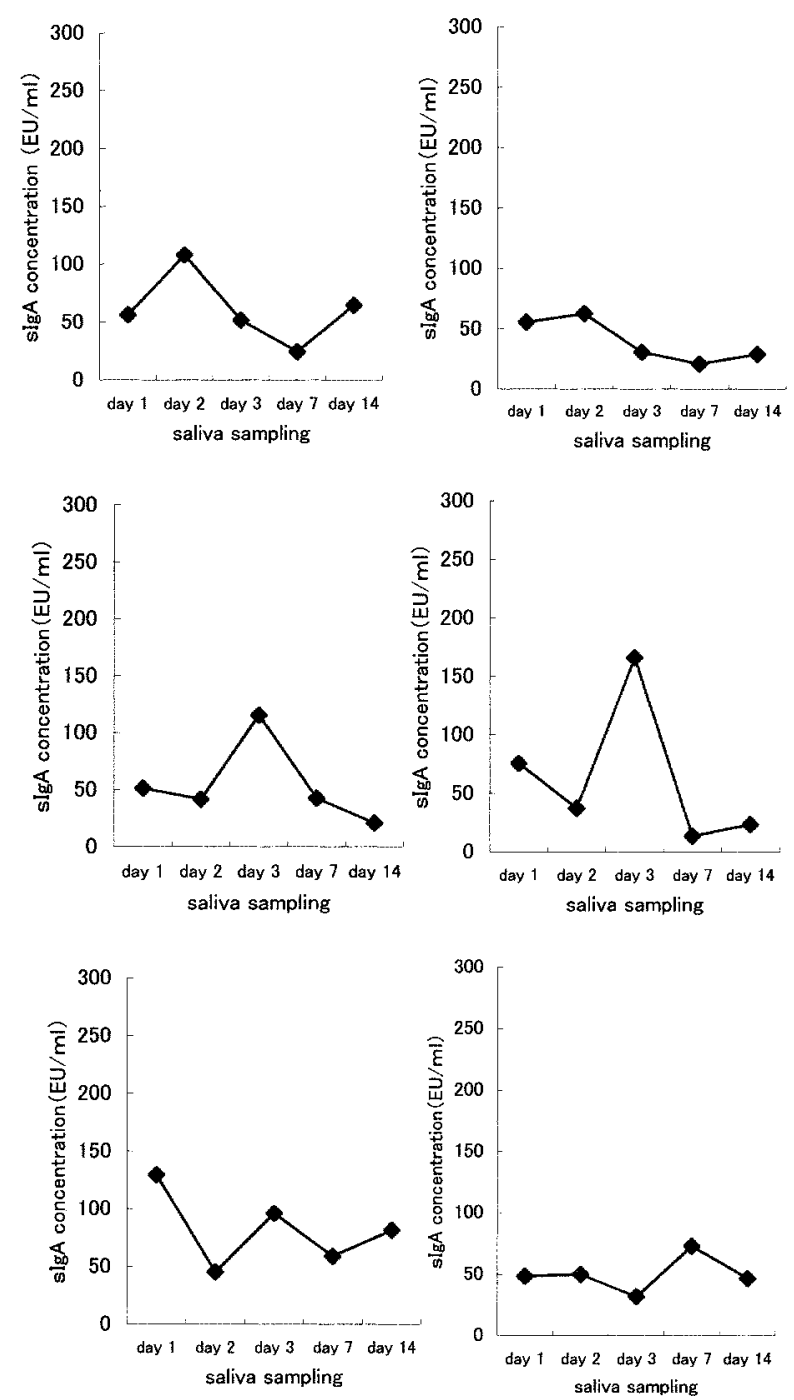

Fig. 3. Variation in sIgA concentration in inferior dogs during the estimation period.

Table 1. Trainers' test and ability as guide dogs

\begin{tabular}{cccc}
\hline \multirow{2}{*}{ Trainers' test } & \multicolumn{2}{c}{ Ability as guide dogs } & \\
\cline { 2 - 3 } & acceptable(head) & rejected(head) & total \\
\hline suitable & 25 & 22 & 47 \\
unsuitable & 0 & 22 & 22 \\
\hline total & 25 & 44 & 69 \\
\hline
\end{tabular}

were superior, and a 9 were inferior.

The sIgA concentration in acceptable dogs gradually increased from day 1 to day 14 , and the variation was significant $(\mathrm{P}<0.001)$ (Fig. 1). This pattern of sIgA variation was shown in all superior dogs, and the lowest sIgA concentration on day 14 was $151.9 \mathrm{EU} / \mathrm{ml}$ (Fig. 2). On the other hand, the sIgA concentration in inferior dogs remained low, and the highest $\operatorname{sIgA}$ concentration on day 14 was $81.5 \mathrm{EU} / \mathrm{m} l$ (Fig. 3). The difference between $\operatorname{sIgA}$ concentration in acceptable dogs and that in rejected dogs was significant on day $14(\mathrm{P}<0.001)$ (Fig. 1).

The parameters for evaluating the efficiency of the trainer's test showed sensitivity was $100 \%$, specificity was $50 \%$, positive predictive value was $46.8 \%$, negative predictive value was $100 \%$, accuracy was $68.1 \%$, likelihood ratio was 2.0 , and diagnostic efficiency was $50 \%$ (Table 1 ).

The cut-off point of sIgA test was $90 \mathrm{EU} / \mathrm{ml}$, and the sensitivity was $92.0 \%$, specificity was $70.4 \%$, positive predictive value was $63.8 \%$, negative predictive value was $93.9 \%$, accuracy was 78.3 , likelihood ratio was 3.1 , and diagnostic efficiency was $64.8 \%$ (Fig. 4).

Regarding the efficacy of the parallel test, sensitivity was $92.0 \%$, specificity was $79.5 \%$, positive predictive value was $71.9 \%$, negative predictive value was $94.6 \%$, accuracy was $84.1 \%$, likelihood ratio was 3.6 , and diagnostic efficiency was $73.1 \%$ (Table 2).

Study 2. Correlation between sIgA concentration during the puppy period and adaptive ability as guide dogs.

Among the 26 dogs, 6 dogs were acceptable and 20 rejected.

No significant differences were found on any sampling day (Fig. 5).

\section{DISCUSSION}

The present study showed that SIgA concentration on day 14 of the estimation period was significantly different between acceptable dogs and rejected dogs. These results are thought to show the potential of salivary sIgA concentration as a marker of adaptive ability in prospective guide dogs.

Although the sensitivity and negative predictive value of the trainer's test were complete $(100 \%)$, the rejection of unsuitable dogs during the estimation period is very important as evaluation is followed by a long period of intense training. On this point, the specificity of sIgA test (70.4\%) was higher than that of trainer's test $(50 \%)$, and this result suggests that more unsuitable dogs could be rejected, if the sIgA test was used. Moreover, using both the trainer's test and $\operatorname{sIgA}$ test as a parallel test giving priority to specificity, specificity was $79.5 \%$, and efficacy was improved. Therefore, combination of the sIgA test with the trainer's test was extremely useful in estimating adaptive ability as guide dogs for the blind.

During the estimation period, $\operatorname{sg}$ A concentration was lowest on day 1 when the prospective guide dogs were separated from their puppy-workers and their environment was changed largely. The low sIgA concentration suggested a stress response, as many studies have shown that sIgA concentration is decreased by stress in human and dogs $[6,7$, $10,12,19]$. A difference in sIgA concentration between superior dogs and inferior dogs gradually became observable, though the value and degree of stimulus were the same in that the prospective guide dogs were housed in the identi- 
Table 2. Parallel test (trainers' test and sIgA test were conducted simultaneously) and ability as guide dogs

\begin{tabular}{cccc}
\hline & \multicolumn{2}{c}{ Ability as guide dogs } & \\
\cline { 2 - 3 } Parallel test & acceptable(head) & rejected(head) & total \\
\hline suitable & 23 & 9 & 32 \\
unsuitable & 2 & 35 & 37 \\
\hline total & 25 & 44 & 69 \\
\hline
\end{tabular}

\section{ROC curve}

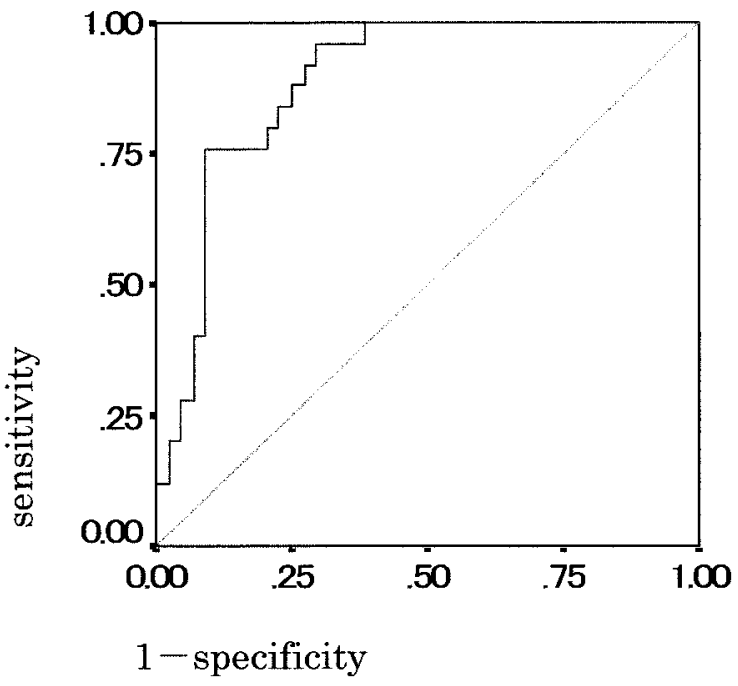

Fig. 4. The receiver operating characteristics curve (ROC curve) for SIgA concentration on day 14 .

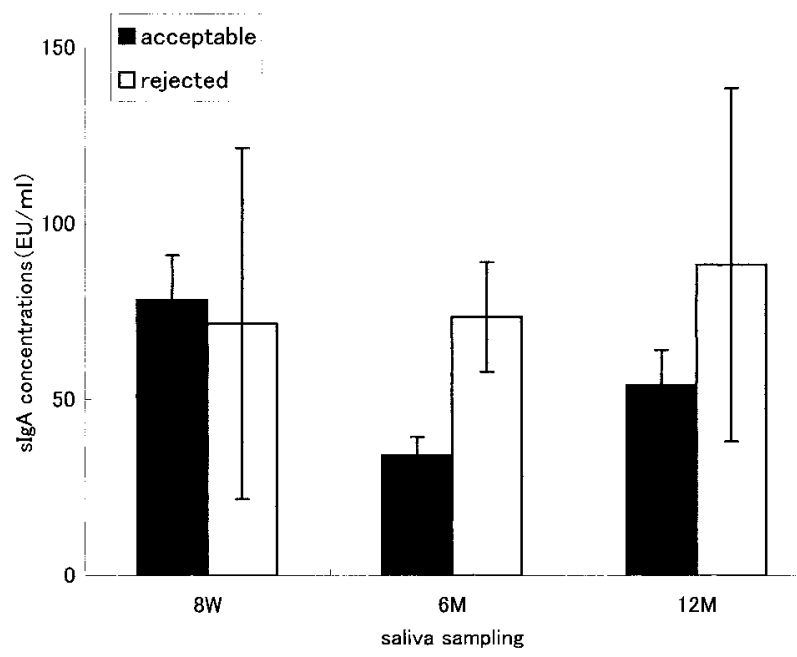

Fig. 5. The sIgA concentration in acceptable dogs $(n=6)$ or rejected dogs $(n=20)$ during the puppy period (average $\pm S E)$. cal kennels and had a same care. Therefore, the sIgA levels during the estimation period indicated sensitivity and adaptability to stress, such as changes in environment and various environmental stimuli, in each dog. Specifically, the dogs whose sIgA concentration was low appear to lack adaptability to stress and be of unstable temperament.

On the other hand, sIgA concentration during the puppy period (during the prospective guide dogs were housed in each puppy raisers' houses) was shown to have no correlation to the adaptive ability of the guide dogs for the blind. In the present study, the saliva samples were collected in the morning, before feeding and walking, because the effects of sampling time, feeding, and activity on salivary $\operatorname{sigA}$ concentration have been reported in dogs and humans $[8,10$, 15]. However, it was impossible to collect saliva understandardized condition and the value and degree of stimuli on each dog was different. Therefore, if standardized condition like the estimation period can be set up for puppies, it may be possible to estimate the adaptability earlier. As earlier estimation is very important and improves the efficiency of training guide dogs, further studies are necessary.

The present study showed that SIgA concentration is an objective marker of the adaptive ability of guide dogs for the blind, and the dogs whose sIgA concentration is lower than $90 \mathrm{EU} / \mathrm{m} l$ are suggested to be unsuitable as guide dogs because of their lack of adaptability to stress. The sIgA test is also suggested to be useful in improving animal welfare. Moreover, the cost and labor involved in raising guide dogs could be reduced.

ACKNOWLEDGMENTS. This research was supported and assisted by the Hokkaido Guide Dogs for the Blind Association. The authors would particularly like to thank Mr. Fumiaki Nagasaki, and the puppy-workers for their excellent assistance and help in saliva collection.

\section{REFERENCES}

1. Deinzer, R., Kleineidam, C., Stiller-Winkler R., Idel, H. and Bachg, D. 2000. Prolonged reduction of salivary immunoglobulin A (sIgA) after a major academic exam. Int. J. Psychophysiol. 7: 219-232.

2. Deinzer, R. and Schuller, N. 1998. Dynamic of stress-related decrease of salivary immunoglobulin $\mathrm{A}(\mathrm{sIg} \mathrm{A})$ : relation to symptoms of common cold and studying behavior. Behav. Med. 23: 161-169.

3. Doddard, M. E. and Beilharz, R. G. 1984. A factor analysis of fearfulness in potential guide dogs. Appl. Amim. Behav. Sci.12: 253-265.

4. Evans, P., Der, G., Ford, G., Hucklebridge, F., Hunt, K. and Lambert, S. 2000. Social class, sex, and age differences in mucosal immunity in a large community sample. Brain.Behav. Immun. 14: 41-48.

5. Goddard, M. E. and Beilharz, R. G. 1982. Genetic and environmental factors affecting the suitability of the dogs as guide dogs for the blind. Theor. Appl. Genet. 62: 97-102.

6. Graham, N. M. H., Bratholomeust, R. C. A., Taboonpong, N. and Brooy, J. T. 1988. Douse anxiety reduce the secretion rate of secretory IgA in saliva? Med. J. Aust.148: 131-133. 
7. Henningsen, G. M., Hurrell, J. J., Baker, F., Douglas, C., Mackenzie, B. A., Robertson, S. K. and Phipps, F. C. 1992. Measurement of salivary immunoglobulin A as an immunologic biomarker of job stress. Scand. J. Work Environ. Health 18: $133-136$.

8. Hucklebridge, F., Clow, A. and Evans, P. 1998, The relationship between salivary secretory immunoglobulin A and cortisol:neuroendocrine response to awakening and the diurnal cycle. Int. J. Psychophysiol. 31: 69-76.

9. Hucklebridge, F., Lambert, A. and Clow, A. 2000. Modulation of secretory immunoglobulin A in saliva; response to manipulation of mood. Biol. Phychol. 53: 25-35.

10. Kikkawa, A., Uchida, Y., Nakade, T. and Taguchi, K. 2003. Salivary secretory IgA concentrations in beagle dogs. J. Vet. Med. Sci. 65: 689-693.

11. Kugler, J., Reintjes, F. and Tewes, V. 1996. Competition stress in soccer coaches increases salivary immunoglobin A and salivary cortisol concentrations. J. Sports Med. Phys. Fitness. 36: $117-120$

12. Lowe, G., Urquhart, J., Greenman, J. and Lowe, G. 2000. Academic stress and secretory immunoglobulin A. Psychol. Rep. 87: 721-722.

13. Martin, R. A. and Dobbin, J. P. 1988. Sense of humour, hassles, and immunoglobulin A:evance for a stress moderating effect of humour. Int. J. Psychiatry Med. 18: 93-105.

14. Ohira, H., Watanabe, Y., Kobayashi, K. and Kawai, M. 1999. The type A behavior pattern and immune reactivity to brief stress: change of volume of secretory immunoglobulin A in saliva. Percept. Mot. Skills. 89: 423-430.

15. Schiffman, S. S. and Miletic, I. D. J. 1999. Effect of taste and smell on secretion rate of salivary IgA in elderly and young persons. J. Nutr. 3: 158-164.

16. Scott, J. P. and Bielfelt, S. W. 1976. Analysis of the puppytesting program. pp. 39-76. In: Guide Dogs for the Blind: Their Selection, Development and Training (Pfaffenberger, C. J., ed.), Elsevier, Amsterdam.

17. Serpell, J. A. and Hsu, Y. 2000. Development and validation of a novel method for evaluating behavior and temparament in guide dogs. Appl. Anim. Behav. Sci. 72: 347-364.

18. Skandakumar, S., Stodulski, G. and Hau, J. 1995. Salivary IgA: A possible stress marker in dogs. Animal Welfare 4: 339-350.

19. Spangler, G. 1997. Psychological and physiological responses during an exam and their relation to personality characteristics. Psychoneuroendocrinology 22: 423-441.

20. Stone, A. A., Neale, J. M., Cox, D. S., Napoli, A., Valdimarsdottie, H. and Keddedy-Moore, E. 1994. Daily events are associated with a secretory immune response to an oral antigen in men. Health. Psychol. 13: 440-446.

21. Willemsen, G., Ring, C., Carroll, D., Evans, P., Clow, A. and Hucklebridge, F. 1998. Secretory immunoglobulin A and cardiovascular reactions to mental arithmetic and cold pressor. Psychophysiology 35: 252-259.

22. Willis, M. B. 1995. Genetic aspects of dog behaviour with particular reference to working ability. pp. 51-64. In: The Domestic Dogs: Its Evolution, Behaviour, and Interaction with People (Serpell, J. ed). Cambridge University Press, New York.

23. Zeier, H., Brauchli, P. and Joller-Jemelka, H.I. 1996. Effects of work demands on immunoglobulin A and cortisol in air traffic controllers. Biol. Psychol. 42: 413-423. 